\title{
Sociologia de Bourdieu: avaliação da produção científica dos pesquisadores de produtividade em pesquisa em Ciência da Informação ${ }^{1}$
}

\author{
Bruno Henrique Alves \\ Doutor; Universidade Federal Fluminense, Niterói, RJ, Brasil; \\ brhenriquealves@id.uff.br \\ Ely Francina Tannuri de Oliveira \\ Doutora; Universidade Estadual Paulista, Marília, SP, Brasil; \\ etannuri@gmail.com
}

\begin{abstract}
Resumo: O campo científico da Ciência da Informação é um espaço hierarquizado de posições e formado por relações objetivas. Esta pesquisa objetivou compreender a geração e construção do conhecimento dos pesquisadores de produtividade em pesquisa em Ciência da Informação. De forma específica, propõe-se a apresentar os pesquisadores de produtividade em pesquisa em Ciência da Informação e agrupar os diferentes artigos produzidos por eles, segundo os temas dos Grupos de Trabalhos da Associação Nacional de Pesquisa e Pós-Graduação em Ciência da Informação. Como procedimento, a população da pesquisa foi constituída por um total de 47 pesquisadores de produtividade em pesquisa em Ciência da Informação, no primeiro quinquênio 2005-2009, e 56, no segundo quinquênio 2010-2014. Utilizou-se um total de 317 artigos completos publicados em periódicos, no primeiro quinquênio, e 597, no segundo quinquênio. Esses artigos foram utilizados para desenvolver os agrupamentos com base nos temas dos 11 Grupos de Trabalhos da Associação Nacional de Pesquisa e Pós-Graduação em Ciência da Informação e para gerar as redes two-mode por meio do software Ucinet. Como resultado, identificou-se que a construção do trabalho científico visa estabelecer um conhecimento adequado para orientar o funcionamento desse trabalho a partir das relações objetivas apresentadas pelos pesquisadores. Como conclusão, pode-se destacar que os pesquisadores de produtividade em pesquisa em Ciência da Informação, envolvidos nesse jogo de poder, têm interesses específicos definidos pela lógica do jogo, ou seja, sempre há competição e disputas por troféus, como, por exemplo, na disputa dos melhores trabalhos, na disputa pela coordenação científica, entre outras.
\end{abstract}

Palavras-chave: Campo científico. Construção do conhecimento. GTs da ANCIB. 


\section{Introdução}

Para compreender a construção do conhecimento científico, destacam-se as contribuições da Sociologia da Ciência, "[..] que estuda os modos pelos quais a pesquisa científica e a difusão do conhecimento científico são influenciadas pelas condições sociais e, por seu turno, influenciam o comportamento social" (BEN-DAVID, 1975, p. 1). A Sociologia da Ciência investiga as relações sociais e, também, as posições no espaço social, "[..] tal como talvez ela possa ser definida a partir de critérios e propriedades exclusivamente universitários esteja tão estreitamente ligada às tomadas de posição [...]" (BOURDIEU, 2013, p. 167) sociais e políticas entre os agentes em um determinado espaço social de poder.

Considerando-se sua relevância e contribuição para a construção do conhecimento nos diferentes campos da ciência, muitas pesquisas foram desenvolvidas, destacando-se as seguintes: Barata e Goldbaum (2003) analisaram o perfil dos pesquisadores de produtividade em pesquisa no campo da Saúde Coletiva; Guimarães, Grácio e Matos (2014) desenvolveram uma pesquisa com o objetivo de analisar a produção científica dos pesquisadores de produtividade em pesquisa no campo da Ciência da Informação (CI); Sacco et al. (2016) desenvolveram um estudo com o objetivo de analisar o perfil dos pesquisadores de produtividade em pesquisa vinculados ao campo da Psicologia; Leite e Rocha Neto (2017) apresentaram um trabalho com o objetivo de analisar o perfil dos pesquisadores de produtividade em pesquisa no campo da Educação; Bufrem, Oliveira e Sobral (2018) sistematizaram um estudo a partir da produção científica indexada na Base de Dados Referenciais de Artigos de Periódicos em Ciência da Informação (BRAPCI) sobre os temas relacionados ao Grupo de Trabalho (GT) 07 da Associação Nacional de Pesquisa e Pós-Graduação em Ciência da Informação (ANCIB), considerando os pesquisadores de produtividade em pesquisa, nível um (1), em CI. Ainda, Melo, Bufrem e Correia (2018) analisaram a produção científica dos pesquisadores de produtividade em pesquisa, nível um (1), em CI, registrada nos anais do Encontro Nacional de Pesquisa em Ciência da Informação (ENANCIB), tendo em vista sua caracterização e participação no evento. 
A partir da criação do Conselho Nacional de Desenvolvimento Científico e Tecnológico (CNPq), em 1951, originou-se a primeira instituição brasileira destinada a estimular a pesquisa científica e tecnológica no contexto da formação de recursos humanos para pesquisa. O CNPq é responsável pela concessão de bolsas - de bolsas no país e no exterior e de bolsas para empresas - e de auxílios de diversas modalidades a pesquisadores advindos dos diferentes campos do conhecimento científico (MUELLER; SANTANA, 2003).

Na classificação adotada pelo CNPq, a CI é um dos campos incluídos na área de Ciências Sociais Aplicadas, na qual se encontram outros campos, como: Administração, Arquitetura da Informação, Comunicação, Demografia, Desenho Industrial, Direito, Economia, Economia Doméstica, Museologia, Planejamento Urbano e Regional, Serviço Social e Turismo (CNPq, 2017). A Arquivologia, a Biblioteconomia e a Teoria da Informação são consideradas subcampos da CI (CNPq, 2017).

O CNPq, agência de fomento, contribuiu para a criação e divulgação das bolsas de Produtividade em Pesquisa (PQ). Essas bolsas surgiram em 1976, voltadas aos vários campos do conhecimento científico e direcionadas aos “[...] pesquisadores que se destaquem [se destacassem] entre seus pares, valorizando sua produção científica segundo critérios normativos, estabelecidos pelo CNPq, e específicos, pelos Comitês de Assessoramento (CAs) do CNPq." (CNPq, 2017, doc. não paginado).

Essas bolsas são classificadas/categorizadas da seguinte forma: sênior (SR), categoria/nível um (1), com quatro níveis A, B, C e D, e categoria/nível dois (2). Espera-se que os pesquisadores enquadrados na categoria/nível um (1) tenham uma importante inserção científica em âmbito nacional e/ou internacional e que os da categoria dois (2) sejam avaliados, principalmente, por meio de sua produção científica e suas orientações (CNPq, 2017).

Para maior elucidação, faz-se necessário sistematizar a formação dos GTs da ANCIB, que vieram sofrendo alterações de nomenclatura. Na discussão realizada pelo Fórum dos Coordenadores de Grupos de Trabalho, ocorrida durante o sexto ENANCIB, realizado no município de Florianópolis (SC), em 30/11/2005 (ANCIB, 2016), assim ficou estabelecido: 
Atualmente, as temáticas presentes na ANCIB (2016) estão organizadas por 11 GTs, a saber: GT-01-Estudos Históricos e Epistemológicos da CI; GT02-Organização e Representação do Conhecimento; GT-03-Mediação, Circulação e Apropriação da Informação; GT-04-Gestão da Informação e do Conhecimento; GT-05-Política e Economia da Informação; GT-06-Informação, Educação e Trabalho; GT-07-Produção e Comunicação da Informação em Ciência, Tecnologia \& Inovação; GT-08-Informação e Tecnologia; GT-09Museu, Patrimônio e Informação; GT-10-Informação e Memória; GT-11Informação \& Saúde.

A partir do exposto, delimita-se a questão de pesquisa: como os artigos produzidos pelos pesquisadores de produtividade em pesquisa em CI contribuem para a construção do conhecimento científico no campo em estudo?

Esta pesquisa objetiva, de forma geral, compreender os mecanismos implícitos na geração e na construção do conhecimento dos pesquisadores de produtividade em pesquisa em CI. De forma específica, propõe-se a apresentar, diacronicamente, os pesquisadores de produtividade em pesquisa em CI e agrupar, por meio dos indicadores bibliométricos, os diferentes artigos produzidos por esses pesquisadores, segundo os temas dos GTs da ANCIB, e desenvolver uma análise a partir da Sociologia de Pierre Bourdieu. Essa abordagem justifica-se em virtude de serem pouco explorados os estudos que objetivam entender a CI a partir dos indicadores bibliométricos e da Sociologia de Pierre Bourdieu. A escolha dos pesquisadores de produtividade em pesquisa em CI baseou-se no fato de que o CNPq tem sido um dos órgãos de fomento mais presentes na história do desenvolvimento da CI (MUELLER; SANTANA, 2003).

\section{Fundamentos: sociologia de Pierre Bourdieu}

Nesta seção o foco central envolve os seguintes conceitos: campo científico, capital cultural, capital científico e indicadores bibliométricos. Eles serão utilizados para fundamentar esta pesquisa. 


\subsection{O campo científico}

Bourdieu $(1975,2008)$ define o campo científico como um espaço social dotado de uma estrutura e de conflitos pela manutenção da posição social que determinado agente e/ou instituição ocupa. É um lugar abstrato, ocupado por dominantes, dominados e pretendentes.

Um campo pode ser definido como

[...] uma rede ou uma configuração de relações objetivas entre posições [ocupadas pelos agentes]. Essas posições estão objetivamente definidas na sua existência e nas determinações que impõem a seus ocupantes, agentes ou instituições, pela sua situação (situ) atual e potencial na estrutura de distribuição das diferentes espécies de poder (ou de capital), cuja possessão comanda o acesso aos benefícios específicos que estão em jogo no campo, e, ao mesmo tempo, pelas suas relações objetivas com as outras posições (dominação, subordinação, homologia, etc.) (BOURDIEU; WACQUANT, 1992, p. 97, tradução nossa).

O campo científico é formado pelos agentes e/ou pelas instituições, aqui personalizados pelos pesquisadores de produtividade em pesquisa em CI, pois nesse espaço há concorrências e também manutenção da posição ocupada em seu interior (BOURDIEU, 1975, 1983, 2008).

\subsection{Capital cultural, capital científico e indicadores bibliométricos}

O capital cultural é mensurado e entendido a partir de algumas variáveis, tais como: título escolar, origem social e frequência em cinema, em concertos e em teatros, entre outras variáveis (BOURDIEU, 1983). O capital cultural apresentase de três formas: capital incorporado (história pessoal), capital objetivado (produção de livros, teses, artigos científicos, capítulos de livros, monumentos, entre outros) e capital institucionalizado (medido pela legitimação por determinado agente) (BOURDIEU, 1979). A estrutura do campo científico é um estado da relação de força entre os diferentes agentes e/ou instituições engajados na luta científica composta pela distribuição do capital científico por aqueles nele inseridos.

Para Bourdieu (2004, 2008), o capital científico está classificado em duas espécies: o "puro, específico ou [relativo a uma] autoridade propriamente científica", que pode ser detectado por meio dos indicadores de citação como, 
por exemplo, o número de citações que determinado agente e/ou instituição recebe, e o "institucional, temporal, político ou [relativo ao] poder sobre o mundo científico", ligado à ocupação de posições importantes nas instituições científicas e/ou na administração científica como, por exemplo, a de coordenador de grupo de pesquisa, a de chefe de departamento, entre outras posições.

O indicador de produção científica ou "[...] indicador de produtividade congrega mecanismos especiais de análise de dados e varia conforme o tipo de metodologia adotada pelo pesquisador que trabalha com estudos bibliométricos" (MUGNAINI; CARVALHO; CAMPANATTI-OSTIZ, 2006, p. 325). Refere-se ao número de documentos produzidos pelos países, instituições e/ou pesquisadores, tais como livros, artigos científicos, capítulos de livros, trabalhos publicados em anais etc. (FAPESP, 2005).

Os indicadores de ligação são utilizados a partir das coocorrências de autoria, citações, cocitações e palavras nos diferentes campos científicos e são aplicados para gerar as redes de colaboração científica e/ou relacionamentos e os mapas estruturais de conhecimento entre os diferentes agentes e/ou instituições a partir de procedimentos estatísticos e matemáticos objetivando a visualização dos agrupamentos.

Os indicadores de citação baseiam-se no número de citações que determinado documento ou pesquisador recebe em determinada época e mostram o impacto e/ou a visibilidade dentro de determinado campo científico (FAPESP, 2005).

Inicialmente, para o desenvolvimento e sistematização desta pesquisa, utilizaram-se somente os indicadores de produção e ligação, tendo em vista os indicadores bibliométricos para a apresentação e análise dos dados.

\section{Procedimentos metodológicos}

Esta pesquisa apresenta como características aspectos relacionados aos métodos quantitativos e qualitativos, ao método documental e ao método bibliográfico, principalmente os indicadores bibliométricos que envolvem a produção e a ligação presentes na produção científica ora investigada. Em setembro de 2015, 
buscaram-se os pesquisadores de produtividade em pesquisa em CI, no período de 2005 a 2009 e de 2010 a 2014, divididos em dois quinquênios, a partir do site do CNPq (2019a).

Acessou-se o item serviços - dados abertos - histórico bolsas/ano e recuperaram-se os arquivos em Extensible Markup Language (XML), nos quais foram encontradas as seguintes informações: ano-pagamento; nome; sexo; modalidade do processo; categoria-nível-da-modalidade; data-início-processo; data-término-processo; número-meses-pagos; valor-pago; nome-instituição; nome-da-área-do-conhecimento; título do processo, entre outras informações (CNPq, 2015).

Com base nesse levantamento, a população da pesquisa foi constituída por um total de 47 pesquisadores de produtividade em pesquisa em CI, no primeiro quinquênio 2005-2009, e 56 no segundo quinquênio 2010-2014.

Em seguida, buscou-se o Currículo Lattes ${ }^{2}$ de cada pesquisador por meio da plataforma Lattes para verificar, recuperar e organizar a produção científica relativa a artigos científicos publicados em periódicos. Identificaram-se 327 artigos, nesse primeiro quinquênio, publicados pelos 47 pesquisadores de produtividade em pesquisa, considerando-se que a quantidade de pesquisadores em cada ano foi diferente; ou seja, em um ano específico, alguns deles não foram pesquisadores de produtividade em pesquisa, o que tornou seu período descontínuo, embora isso não estivesse explicitado nos dados. Estes detalhes e particularidades também se manifestaram, no período de 2010 a 2014. Observou-se o nível/categoria de cada pesquisador PQ para verificar se o pesquisador produziu artigos no período em estudo e, se produziu, para medir a quantidade de artigos publicados por ano. Também se verificou se havia duplicação de artigos decorrente da coautoria entre os pesquisadores de produtividade em pesquisa em CI; ou seja, buscou-se verificar se, a partir do momento em que dado artigo foi publicado de 2005 a 2009 e/ou de 2010 a 2014, cada autor registrou essa publicação em seu próprio Currículo Lattes. Nesse sentido, por conta da duplicação de informação, o artigo foi somado somente uma vez ao total. A partir do total de 327 artigos, retiraram-se as duplicações, e 
isso produziu um total de 317 artigos completos publicados em periódicos. Essas duplicações foram retiradas por meio dos recursos do Microsoft Word.

Em seguida, buscaram-se os pesquisadores de produtividade em pesquisa em CI, no período de 2010 a 2014. Utilizaram-se as mesmas variáveis do primeiro quinquênio. Com base nesse levantamento, a população da pesquisa foi constituída por 56 pesquisadores de produtividade em pesquisa em CI, no segundo quinquênio. A partir do total de 637 artigos, retiraram-se as duplicações, o que reduziu essa soma a um total de 597 artigos completos publicados em periódicos. Essas duplicações também foram retiradas por meio dos recursos do Microsoft Word.

Os 317 artigos produzidos no primeiro quinquênio e os 597 artigos produzidos no segundo quinquênio foram agrupados com base nos temas (ementas) dos 11 GTs da ANCIB.

Utilizaram-se o título, o resumo e as palavras-chave para categorizar os artigos em uma ou mais ementas dos GTs (não mutuamente exclusivas, ou seja, o artigo poderia estar relacionado em mais de um GT da ANCIB). Com isso, o número total de artigos categorizados não reflete o número total de artigos identificados, coletados e utilizados para o desenvolvimento desta pesquisa, considerando-se que um mesmo artigo pode estar classificado em mais de uma categoria. A seguir, procedeu-se a análise, destacando-se os GTs mais frequentes. Com isso, os pesquisadores de produtividade em pesquisa também foram associados, dependendo do caso, a mais de um dos GTs da ANCIB.

Esses características também podem ser identificadas no trabalho intitulado Cenário e perspectiva da produção científica sobre competência em informação (CoInfo) no Brasil: estudo da produção no âmbito da ANCIB. Nessa pesquisa, “[...] foram analisados em cada comunicação oral os respectivos títulos, palavras-chave, resumos e também o texto completo." (LEITE et al., 2016, p. 155). Ainda, Noronha e outros (2007) analisaram a temática de teses defendidas sobre CI, a partir dos temas do GTs da ANCIB.

Posteriormente, geraram-se as redes de relações entre as duas variáveis: primeiro quinquênio, a partir da matriz 47x11 (pesquisadores e GTs da ANCIB); segundo quinquênio, a partir da matriz 56x11 (pesquisadores e GTs da ANCIB). 
Essas matrizes foram construídas por meio do Microsoft Excel e utilizou-se o software Ucinet para gerar as redes de relações two-mode ${ }^{3}$ entre as duas variáveis, portanto, de forma automatizada.

A partir da coleta, organização e tratamento dos dados, utilizou-se a Sociologia da Ciência, especialmente a Sociologia de Pierre Bourdieu (2008), que tem como elemento que "Um cientista é a materialização de um campo científico e as suas estruturas cognitivas são homólogas à estrutura do campo e, por isso, constantemente ajustadas às expectativas inscritas no campo" (BOURDIEU, 2008, p. 62).

\section{Apresentação e análise dos dados}

Retome-se Bourdieu (1975, 2008), com o conceito de campo científico como um contexto que apresenta uma estrutura e um espaço social e também relações objetivas, considerando as peculiaridades da produção do conhecimento científico.

Além disso, esse espaço é dinâmico e se fortalece a partir das habilidades e responsabilidades dos agentes e/ou instituições que o constituem. A noção de campo mostra que a CI é uma construção social, coletiva, pois envolve um processo sistematizado dentro de um contexto e época, um campo de forças, lutas e conflitos entre os diferentes agentes e/ou instituições inseridos nesse espaço social, que almejam uma relativa autonomia e reconhecimento e/ou notoriedade dos pares no âmbito brasileiro e estrangeiro (internacional).

Nas Tabelas 1 e 2, apresenta-se a quantidade de pesquisadores de produtividade em pesquisa em CI, e se consideram o primeiro 2005-2009 e segundo 2010-2014 quinquênios, respectivamente. De acordo com a Tabela 1, trabalhou-se com 47 pesquisadores e observou-se, de 2005 a 2009, uma distribuição de um conjunto de dados (número de pesquisadores), ou seja, uma variabilidade no total, em cada ano, bem como nas diferentes categorias/níveis, em virtude da mobilidade dos pesquisadores ocorrida em relação às duas variáveis citadas. Computou-se um total de 47 nomes distintos nas diversas categorias/níveis, durante o quinquênio. O ano de 2007 apresenta-se como outlier, discrepando na curva crescente do período. 
Tabela 1 - Quantidade de pesquisadores de produtividade em pesquisa em Ciência da Informação por categoria/nível e ano de forma inclusiva, no período de 2005 a 2009.

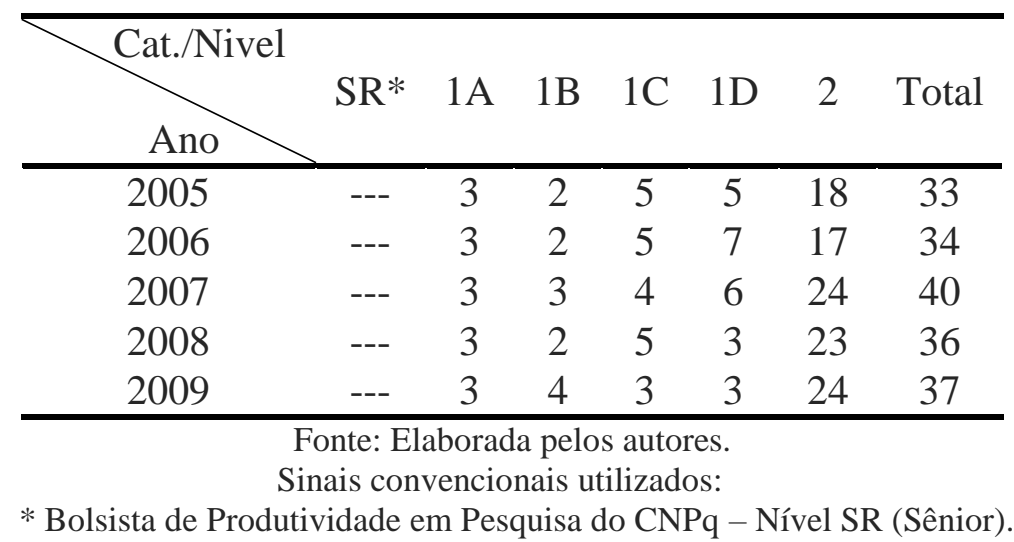

De acordo com a Tabela 2, trabalhou-se com 56 pesquisadores e observou-se, de 2010 a 2014, uma variabilidade nesse total em cada ano, bem como nas diferentes categorias/níveis, em virtude da mobilidade ocorrida em relação às duas variáveis citadas.

Tabela 2 - Quantidade de pesquisadores de produtividade em pesquisa em Ciência da Informação por categoria/nível e ano de forma inclusiva, no período de 2010 a 2014.

\begin{tabular}{cccccccc}
\hline Cano & SR* & $1 \mathrm{~A}$ & $1 \mathrm{~B}$ & $1 \mathrm{C}$ & $1 \mathrm{D}$ & 2 & Total \\
\hline 2010 & ---- & 3 & 3 & 5 & 3 & 28 & 42 \\
2011 & 1 & 4 & 3 & 4 & 3 & 31 & 46 \\
2012 & 1 & 4 & 5 & 2 & 5 & 31 & 48 \\
2013 & 1 & 3 & 5 & 2 & 8 & 32 & 51 \\
2014 & 1 & 3 & 5 & 4 & 8 & 27 & 48 \\
\hline \multicolumn{7}{c}{ Fonte: Elaborada pelos autores. } \\
* Bolsista de Produtividade convencionais utilizados: \\
\multicolumn{10}{c}{ Cesquisa do CNPq - Nível SR (Sênior). }
\end{tabular}

Neste quinquênio, registra-se um crescimento leve e contínuo no total de pesquisadores, com destaque para o níveis 1B e 1D. Os demais dados foram apresentados e analisados de acordo com os objetivos propostos, de modo a apresentar, de forma sincrônica, as publicações na tipologia de artigos publicados pelos pesquisadores de produtividade em pesquisa em CI. 
Agruparam-se os artigos produzidos pelos pesquisadores bolsistas, segundo os temas dos GTs da ANCIB, e foi construída a rede pesquisador/GT para cada quinquênio.

Apresenta-se uma rede two-mode no Grafo 1 na qual se relacionam por meio dos artigos publicados os 47 pesquisadores de produtividade em pesquisa em CI, do primeiro quinquênio de 2005 a 2009, representados por círculos vermelhos, e os GTs da ANCIB, representados por quadriláteros azuis. A espessura dos segmentos que unem o pesquisador ao GT corresponde à frequência com que ele produziu no tema do GT.

O GT-02 é o mais contemplado, refere-se à Organização e Representação do Conhecimento e compreende os seguintes temas: organização e preservação de documentos; ambiências informacionais; representação do conhecimento, entre outros (ANCIB, 2016). Neste GT da ANCIB, foram categorizados 99 artigos, elaborados por 20 do total de 47 pesquisadores de produtividade em pesquisa em CI. Com isso, compreende-se que a grande preocupação dos pesquisadores de produtividade em pesquisa em CI são, principalmente, aspectos da Organização da Informação e da Organização do Conhecimento, alusivas às questões fundantes da CI.

Destaca-se que o GT-08 - Informação e Tecnologia - compreende os seguintes temas: tecnologias da comunicação e informação; representação, armazenamento e recuperação da informação, entre outros (ANCIB, 2016). Foram encontrados 65 artigos enquadráveis nesse GT da ANCIB, considerando o total de 47 pesquisadores de produtividade em pesquisa em CI. Foram 18 os pesquisadores responsáveis por artigos nesse GT em estudo.

No GT-04 - Gestão da Informação e do Conhecimento -, destacam-se os seguintes temas: recursos informacionais; fluxos de informação; gestão do conhecimento e informação; aprendizagem organizacional, entre outros (ANCIB, 2016). Foram encontrados 42 artigos, sendo 11 os pesquisadores responsáveis por eles. Esses pesquisadores também possuem artigos em outros GTs da ANCIB: GT-01, GT-02, GT-03, GT-05, GT-06, GT-07, GT-08 e GT-11. 
Grafo 1 - Rede two-mode 2005-2009 formada pelos pesquisadores de produtividade em pesquisa em Ciência da Informação e por GTs da ANCIB.

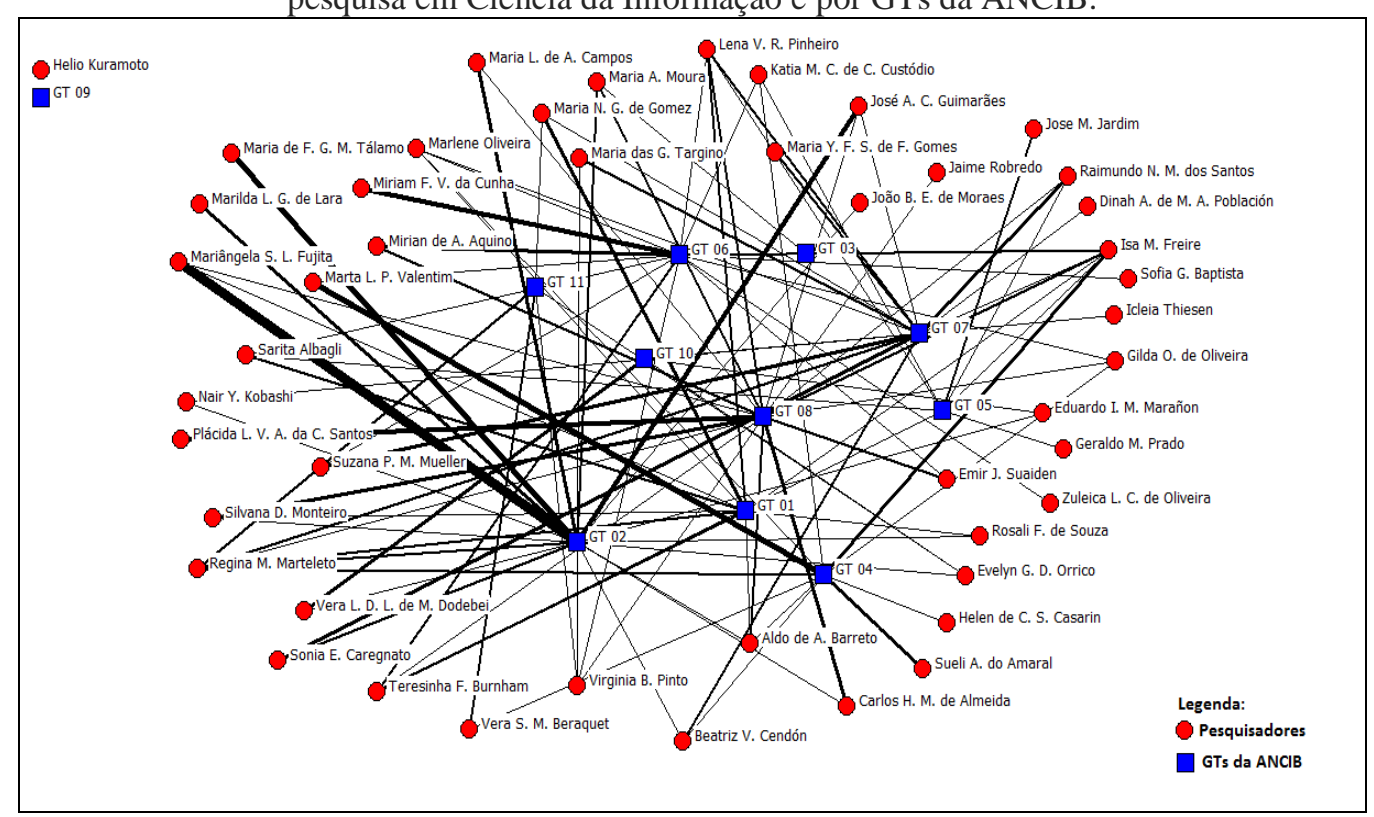

Fonte: Elaborado pelos autores.

O GT-06 - Informação, Educação e Trabalho -, de modo geral, aborda as seguintes temáticas: competências e habilidades; unidades de informação; sociedade do conhecimento; ética nas profissões da informação, entre outras (ANCIB, 2016). Foram encontrados 40 artigos, sendo 15 pesquisadores responsáveis por eles. Esses pesquisadores também possuem artigos em outros GTs da ANCIB: GT-01, GT-04, GT-05, GT-07, GT-08 e GT-11.

O GT-01 - Estudos Históricos e Epistemológicos da Ciência da Informação - compreende os seguintes temas: estudos históricos e epistemológicos; interdisciplinaridade; transdisciplinaridade, entre outros (ANCIB, 2016). Foram encontrados 28 artigos, sendo 11 pesquisadores responsáveis por eles. Esses pesquisadores também possuem artigos em outros GTs da ANCIB: GT-02, GT-04, GT-05, GT-06, GT-07, GT-08 e GT 11.

O GT-05 - Política e Economia da Informação - compreende os seguintes temas: regimes de informação; acesso à informação; política da informação e comunicação; ética da informação; informação e ecologia, entre outros (ANCIB, 2016). Foram encontrados 14 artigos, sendo oito (8) pesquisadores responsáveis por eles. Esses pesquisadores também possuem artigos em outros GTs da ANCIB: GT-01, GT-04, GT-06, GT-07 e GT-08. 
O GT-10 - Informação e Memória - compreende os seguintes temas: campos de conhecimento; memória social; coleções e colecionismo; representações sociais e conhecimento, entre outros (ANCIB, 2016). Foram encontrados 10 artigos, sendo quatro (4) pesquisadores responsáveis por eles. Esses pesquisadores também possuem artigos em outro GT da ANCIB: GT-02 e GT-11.

O GT-11 - Informação \& Saúde - aborda os seguintes temas: inovação em saúde; impacto da informação; políticas de informação em saúde, entre outros (ANCIB, 2016). Foram encontrados 10 artigos, sendo cinco (5) pesquisadores responsáveis por eles. Esses pesquisadores também possuem artigos em outros GTs da ANCIB: GT-01, GT-02, GT-04, GT-06, GT-07; GT08 e GT-10. A participação de um mesmo autor em vários GTs sugere que a CI é uma construção social, coletiva e conjunta, pois envolve um processo sistematizado dentro de um contexto e de uma época, em um campo de forças, lutas e conflitos entre os diferentes agentes.

Por fim, o GT-03 - Mediação, Circulação e Apropriação da Informação - aborda os seguintes temas: mediação e apropriação da informação; dimensões inter e transdisciplinares, entre outros (ANCIB, 2016). Foi categorizado somente um artigo, elaborado por Maria A. Moura.

Apresenta-se na Tabela 3, referente ao primeiro quinquênio de 2005 a 2009, o número de artigos em cada GT da ANCIB associados às diferentes categorias/níveis dos pesquisadores de produtividade em pesquisa.

Dezoito artigos publicados pelos pesquisadores enquadrados na categoria/nível 1A estão associados a seis (6) diferentes GTs da ANCIB: GT-01, GT-02, GT-04, GT-07, GT-08 e GT-11, com especial destaque ao GT-01 e GT08. Identifica-se que 24 artigos publicados pelos pesquisadores enquadrados na categoria/nível 1B estão associados a seis (6) diferentes GTs da ANCIB: GT-01, GT-02, GT-04, GT-07, GT-08 e GT-11.

Quarenta artigos publicados pelos pesquisadores enquadrados na categoria/nível 1C estão associados a sete (7) diferentes GTs da ANCIB: GT-01, GT-02, GT-04, GT-05, GT-06, GT-07 e GT-08. 
Destaca-se que 42 artigos publicados pelos pesquisadores enquadrados na categoria/nível 1D estão associados a sete (7) diferentes GTs da ANCIB: GT01, GT-02, GT-04, GT-05, GT-06, GT-07 e GT-08. Os GTs da ANCIB que apresentam os maiores valores referentes ao número de artigos, em valores absolutos, respectivamente, foram: GT-06, com dez artigos, e GT-02 e GT-04 com nove artigos cada, ou seja, 66,67\%, em valores relativos, dos artigos publicados por pesquisadores de produtividade em pesquisa classificados na categoria/nível 1D.

Tabela 3 - Número de artigos em cada GT da ANCIB associados às diferentes categorias/níveis dos pesquisadores de produtividade em pesquisa, no primeiro quinquênio 2005-2009

\begin{tabular}{|c|c|c|c|c|c|c|c|c|c|c|}
\hline \multirow{3}{*}{$\begin{array}{l}\text { GTs da } \\
\text { ANCIB }\end{array}$} & \multicolumn{10}{|c|}{$\begin{array}{c}\text { Categorias/níveis dos pesquisadores de produtividade em pesquisa e o número de } \\
\text { artigos }\end{array}$} \\
\hline & \multicolumn{2}{|c|}{$\mathbf{1 A}$} & \multicolumn{2}{|c|}{ 1B } & \multicolumn{2}{|c|}{$1 \mathrm{1C}$} & \multicolumn{2}{|c|}{ 1D } & \multicolumn{2}{|r|}{2} \\
\hline & Abs.*** & Rel.**** & Abs. & Rel. & Abs. & Rel. & Abs. & Rel. & Abs. & Rel. \\
\hline 01 & 9 & 50,00 & 6 & 25,00 & 2 & 5,00 & 3 & 7,14 & 8 & 3,58 \\
\hline 02 & 2 & 11,11 & 3 & 12,50 & 13 & 32,50 & 9 & 21,42 & 67 & 30,04 \\
\hline 03 & ----- & ----- & ----- & ----- & ----- & ----- & ----- & ----- & 1 & 0,44 \\
\hline 04 & 1 & 5,55 & 2 & 8,33 & 2 & 5,00 & 9 & 21,42 & 28 & 12,55 \\
\hline 05 & ----- & ----- & ----- & ----- & 1 & 2,50 & 3 & 7,14 & 10 & 4,48 \\
\hline 06 & ----- & ----- & ----- & ----- & 2 & 5,00 & 10 & 23,80 & 29 & 13,00 \\
\hline 07 & 1 & 5,55 & 6 & 25,00 & 10 & 25,00 & 2 & 4,76 & 25 & 11,21 \\
\hline 08 & 4 & 22,22 & 1 & 4,16 & 10 & 25,00 & 6 & 14,28 & 43 & 19,28 \\
\hline 09 & ----- & ----- & ----- & ----- & ----- & ----- & ----- & ----- & ----- & ----- \\
\hline 10 & ----- & ----- & ----- & ----- & ----- & ----- & ----- & ----- & 9 & 4,03 \\
\hline 11 & 1 & 5,55 & 6 & 25,00 & ----- & ----- & ----- & ----- & 3 & 1,34 \\
\hline Total $* * * *$ & 18 & $\sim 100,00$ & 24 & $\sim 100,00$ & 40 & 100,00 & 42 & $\sim 100,00$ & 223 & $\sim 100,00$ \\
\hline & & & & $\begin{array}{r}\text { e: Elaborad } \\
\text { is convencic } \\
* * \mathrm{Abs} \\
* * * \mathrm{Re}\end{array}$ & $\begin{array}{l}\text { pelos } \\
\text { ais ut } \\
\text { luto. } \\
\text { ivo. }\end{array}$ & $\begin{array}{l}\text { itores. } \\
\text { zados: }\end{array}$ & & & & \\
\hline
\end{tabular}

Observa-se que 223 artigos publicados pelos pesquisadores na categoria/nível 2 estão associados a dez (10) diferentes GTs da ANCIB: GT-01, GT-02, GT-03, GT-04, GT-05, GT-06, GT-07, GT-08, GT-10 e GT-11. Os GTs da ANCIB que apresentaram os maiores valores referentes ao número de artigos, em valores absolutos, respectivamente, foram GT-02, com 67 artigos, e GT-08, com 43 artigos; ou seja, 49,32\%, em valores relativos, dos artigos publicados pelos pesquisadores em destaque na categoria/nível 2 estão associados a esses dois GTs apresentados, havendo maior porcentagem de 
artigos no GT-02, que concentra 30,04\%, em valores relativos, dos artigos associados.

Apresenta-se uma rede two-mode no Grafo 2 na qual se relacionam por meio dos artigos publicados os 56 pesquisadores de produtividade em pesquisa em CI, do segundo quinquênio 2010-2014, representados por círculos vermelhos, e os GTs da ANCIB, representados por quadriláteros azuis. O GT-08 da ANCIB é o mais contemplado; nesse GT, foram sistematizadas 154 pesquisas, elaboradas e divulgadas por 28 do total de 56 pesquisadores de produtividade em pesquisa em CI.

Com isso, compreende-se que a grande preocupação dos pesquisadores de produtividade em pesquisa em CI são, principalmente, os estudos teóricos e aplicados para o aperfeiçoamento e utilização dos procedimentos tecnológicos. Esses pesquisadores também possuem artigos em outros GTs da ANCIB: GT01, GT-02, GT-03, GT-04, GT-05, GT-06, GT-07, GT-09, GT-10 e GT-11.

Ao GT-02, foram atribuídos 142 artigos, considerando o total de 56 pesquisadores de produtividade em pesquisa em CI, sendo 28 os responsáveis por artigos nesse tema. Suas pesquisas estão também categorizadas nos seguintes GTs da ANCIB: GT-01, GT-03, GT-04, GT-05, GT-06, GT-07, GT08, GT-09, GT-10 e GT-11.

O GT-07 - Produção e Comunicação da Informação em Ciência, Tecnologia \& Inovação - , por sua vez, compreende os seguintes temas: estudos teóricos; avaliação da produção científica; indicadores científicos, entre outros (ANCIB, 2016). Foram encontrados 142 artigos, sendo 22 pesquisadores responsáveis por eles. Esses pesquisadores também possuem artigos em outros GTs da ANCIB: GT-01, GT-02, GT-03, GT-04, GT-05, GT-06, GT-08, GT-09, GT-10 e GT-11.

No GT-04 foram categorizados 96 artigos, sendo 19 os pesquisadores responsáveis por eles. Esses pesquisadores também têm artigos em outros GTs da ANCIB: GT-01, GT-02, GT-03, GT-05, GT-06, GT-07, GT-08, GT-09, GT10 e GT-11.

No GT-01 foram sistematizados 54 artigos, sendo 24 os pesquisadores responsáveis por eles. Esses pesquisadores também têm artigos em outros GTs 
da ANCIB: GT-02, GT-03, GT-04, GT-05, GT-06, GT-07, GT-08, GT-09, GT10 e GT-11.

No GT-06 foram sistematizados 52 artigos, sendo 20 pesquisadores responsáveis por eles. Esses pesquisadores também têm artigos em outros GTs da ANCIB: GT-01, GT-02, GT-03, GT-04, GT-05, GT-07, GT-08, GT-09, GT10 e GT-11.

No GT-10 foram sistematizados 44 artigos. Os pesquisadores que os escreveram também têm artigos em outros GTs da ANCIB: GT-01, GT-02, GT03, GT-04, GT-05, GT-06, GT-07, GT-08, GT-09 e GT-11.

Grafo 2 - Rede two-mode 2010-2014 formada pelos pesquisadores de produtividade em pesquisa em Ciência da Informação e GTs da ANCIB

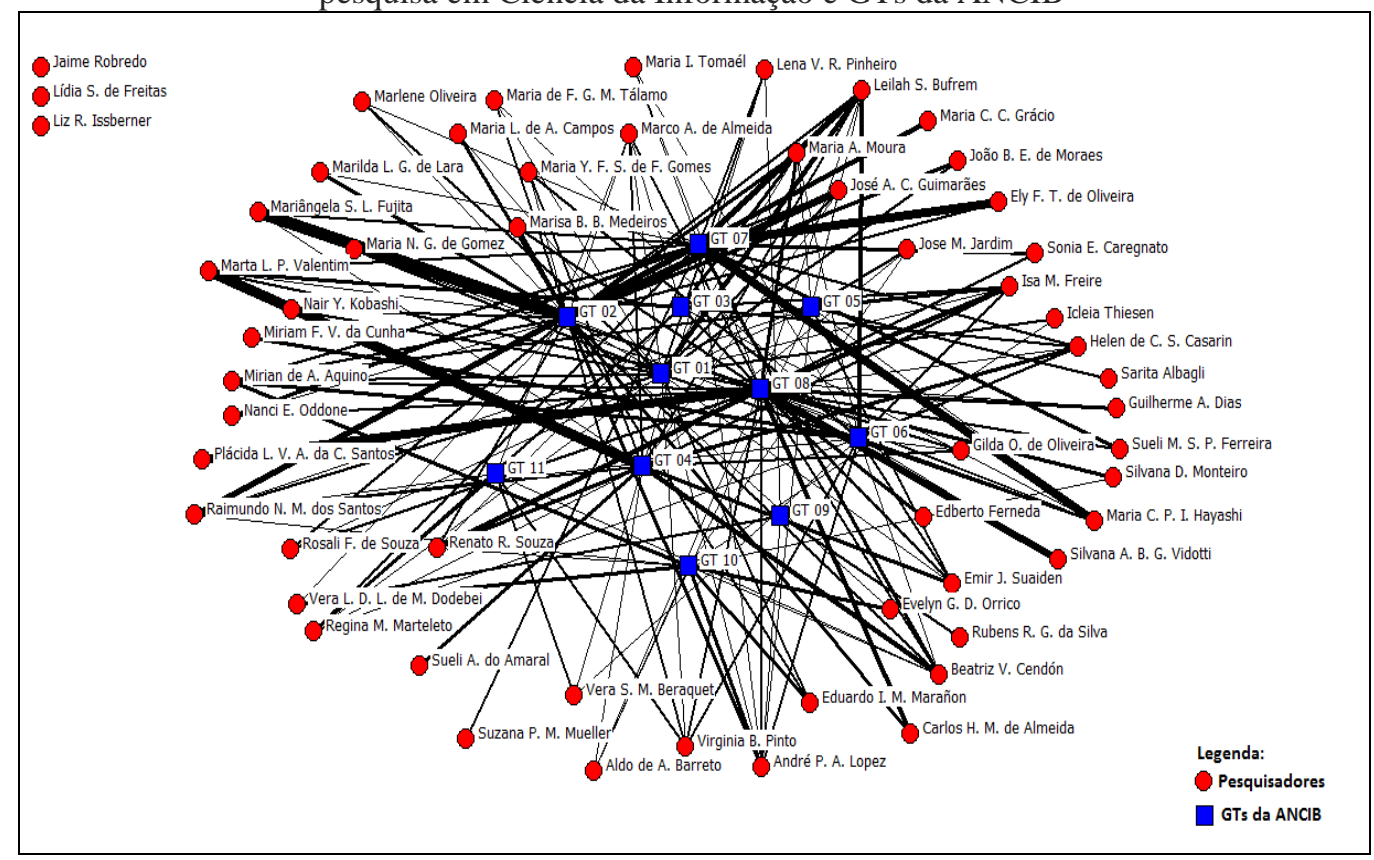

Fonte: Elaborado pelos autores.

Destaca-se também que os pesquisadores que escreveram artigos associados ao GT-03 também têm artigos em outros GTs da ANCIB: GT-01, GT-02, GT-04, GT-05, GT-06, GT-07, GT-08, GT-10 e GT-11, e os pesquisadores que escreveram artigos associados ao GT-05 também têm artigos em outros GTs da ANCIB: GT-01, GT-02, GT-03, GT-04, GT-06, GT-07, GT08, GT-09, GT-10 e GT-11.

Por fim, o GT-11 - Informação \& Saúde - e o GT-09 - Museu, Patrimônio e Informação -, os menos contemplados, abordam os seguintes 
temas: inovação em saúde; impacto da informação; políticas de informação em saúde, entre outros e fenômeno cultural; aspectos informacionais e comunicacionais, entre outros (ANCIB, 2016). Foram sistematizados, respectivamente, 12 artigos e seis (6) artigos em cada GT, porém com pesquisadores dos outros GTs.

Apresenta-se na Tabela 4, referente ao segundo quinquênio de 2010 a 2014, o número de artigos em cada GT da ANCIB associados às diferentes categorias/níveis dos pesquisadores de produtividade em pesquisa.

Os quatro artigos publicados pelo único pesquisador na categoria/nível SR, durante o segundo quinquênio 2010-2014, estão associados a quatro diferentes GTs da ANCIB: GT-04, GT-05, GT-08 e GT-10. A cada GT desses está associado somente um artigo, ou seja, 25,00\%, em valores relativos, dos artigos publicados pelo pesquisador.

Verifica-se que 17 artigos publicados pelos pesquisadores enquadrados na categoria/nível 1A estão associados a seis (6) diferentes GTs da ANCIB: GT01, GT-03, GT-04, GT-06, GT-07 e GT-11. Os GTs da ANCIB que apresentam os maiores valores referentes ao número de artigos, em valores absolutos, respectivamente, foram: GT-07, com seis artigos, e GT-01 e GT-03, ambos com três artigos; ou seja, 70,59\%, em valores relativos, dos artigos publicados pelos pesquisadores em destaque estão associados a esses três GTs apresentados, havendo maior porcentagem de artigos no GT-07, que concentra 35,29\%, em valores relativos, do total de artigos associados.

Tabela 4 - Número de artigos em cada GT da ANCIB associados às diferentes categorias/níveis dos pesquisadores de produtividade em pesquisa, no segundo quinquênio 2010-2014

\begin{tabular}{|c|c|c|c|c|c|c|c|c|c|c|c|c|}
\hline \multirow{3}{*}{$\begin{array}{l}\text { GTs da } \\
\text { ANCIB }\end{array}$} & \multicolumn{12}{|c|}{ Categorias/níveis dos pesquisadores de produtividade em pesquisa e o número de artigos } \\
\hline & \multicolumn{2}{|c|}{ SR* } & \multicolumn{2}{|c|}{$1 \mathbf{A}$} & \multicolumn{2}{|c|}{$1 \mathrm{~B}$} & \multicolumn{2}{|c|}{$1 \mathrm{C}$} & \multicolumn{2}{|c|}{ 1D } & \multicolumn{2}{|c|}{2} \\
\hline & Abs.** & Rel.**** & Abs. & Rel. & Abs. & Rel. & Abs. & Rel. & Abs. & Rel. & Abs. & Rel. \\
\hline 01 & ----- & ----- & 3 & 17,64 & 4 & 10,81 & 7 & 9,45 & 3 & 5,08 & 37 & 6,82 \\
\hline 02 & ----- & ----- & ----- & ----- & 12 & 32,43 & 48 & 64,86 & 4 & 6,77 & 75 & 13,83 \\
\hline 03 & ----- & ----- & 3 & 17,64 & ----- & ----- & 2 & 2,70 & ----- & ----- & 25 & 4,61 \\
\hline 04 & 1 & 25,00 & 1 & 5,88 & 4 & 10,81 & 5 & 6,75 & 17 & 28,81 & 68 & 12,54 \\
\hline 05 & 1 & 25,00 & ----- & ----- & 2 & 5,40 & 4 & 5,40 & 3 & 5,08 & 13 & 2,39 \\
\hline 06 & ----- & ----- & 2 & 11,76 & 4 & 10,81 & ----- & ----- & 7 & 11,86 & 37 & 6,82 \\
\hline 07 & ----- & ----- & 6 & 35,29 & 4 & 10,81 & 5 & 6,75 & 13 & 22,03 & 104 & 19,18 \\
\hline
\end{tabular}




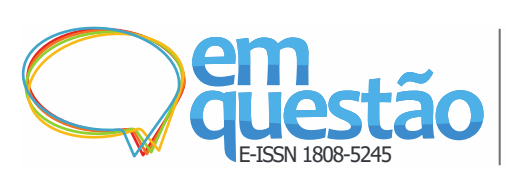

Sociologia de Bourdieu: avaliação da produção científica dos pesquisadores de produtividade em

pesquisa em Ciência da Informação

Bruno Henrique Alves, Ely Francina Tannuri de Oliveira

\begin{tabular}{|c|c|c|c|c|c|c|c|c|c|c|c|c|}
\hline 08 & 1 & 25,00 & ----- & ----- & 4 & 10,81 & 3 & 4,05 & 9 & 15,25 & 128 & 23,61 \\
\hline 09 & ----- & ----- & ----- & ----- & 1 & 2,70 & ----- & ----- & ----- & ----- & 5 & 0,92 \\
\hline 10 & 1 & 25,00 & ----- & ----- & ---- & ----- & ----- & ----- & 2 & 3,38 & 42 & 7,74 \\
\hline 11 & ----- & ----- & 2 & 11,76 & 2 & 5,40 & ----- & ----- & 1 & 1,69 & 8 & 1,47 \\
\hline Total***** & 4 & 100,00 & 17 & $\sim 100,00$ & 37 & $\sim 100,00$ & 74 & $\sim 100,00$ & 59 & $\sim 100,00$ & 542 & $\sim 100,00$ \\
\hline & & & sist & $\begin{array}{r}\text { Fonte } \\
\text { Sin } \\
\text { Produtiv }\end{array}$ & $\begin{array}{r}\text { de em } \\
* * \mathrm{~A} \\
* * * \mathrm{R}\end{array}$ & $\begin{array}{l}\text { o pelos aute } \\
\text { cionais util } \\
\text { esquisa do } \\
\text { oluto } \\
\text { lativo }\end{array}$ & $\begin{array}{l}\text { S. } \\
\text { dos: } \\
\text { NPq }\end{array}$ & vel SR (s & or). & & & \\
\hline
\end{tabular}

Com destaque, os 542 artigos publicados pelos pesquisadores enquadrados na categoria/nível 2 estão associados a todos os GTs da ANCIB, especialmente ao GT-08 $(23,61 \%)$ e ao GT-07 (19,18\%). Esses dois GTs foram os GTs aos quais mais foram associados artigos publicados pelos pesquisadores de produtividade em pesquisa classificados na categoria/nível 2, com os valores absolutos de 128 artigos, no GT-08, e 104 artigos, no GT-07. Esses dois valores totalizam $42,80 \%$ dos artigos publicados por pesquisadores nessa categoria.

A partir dos dados e informações apresentados, identificou-se a composição de pesquisadores exclusivos, ou seja, aqueles que apareceram em somente um GT, no primeiro e no segundo quinquênios. Os pesquisadores do primeiro quinquênio 2005-2009 são: Dinah A. de M. A. Población (GT-07); Geraldo M. Prado (GT-05); Helen de C. S. Casarin (GT-04); Icleia Thiesen (GT-10); Jaime Robredo (GT-08); João B. E. de Moraes (GT-02); José M. Jardim (GT-05); Maria de F. G. M. Tálamo (GT-02); Maria Y. F. S. de F. Gomes (GT-07); Marilda L. G. de Lara (GT-02); Miriam F. V. da Cunha (GT06); Plácida L. V. A. da C. Santos (GT-08); Sofia G. Baptista (GT-06); Sueli A. do Amaral (GT-04) e Zuleica L. C. de Oliveira (GT-06).

Já os pesquisadores do segundo quinquênio 2010-2014 são: Edberto Ferneda (GT-08); Maria C. C. Grácio (GT-07); Miriam F. V. da Cunha (GT-06); Plácida L. V. A. da C. Santos (GT-08); Rubens R. G. da Silva (GT-09); Sarita Albagli (GT-05); Sueli A. do Amaral (GT-04); Suzana P. M. Muller (GT-07).

Os pesquisadores que se mantiveram nos dois quinquênios, tendo em vista os critérios adotados, são: Miriam F. V. da Cunha (GT-06); Plácida L. V. A. da C. Santos (GT-08) e Sueli A. do Amaral (GT-04). 
Nesses agrupamentos, gerados por meio dos indicadores de ligação entre os artigos publicados pelos pesquisadores de produtividade em pesquisa e os temas dos GTs da ANCIB, estão presentes características do campo (conceito que foi definido por Bourdieu combinando elementos presentes na Sociologia de Émile Durkheim, Karl Marx e Max Weber), e esses agrupamentos podem ser relacionados com essas características, considerando suas singularidades, estruturas sociais e propriedades.

No caso desta pesquisa, essa estrutura social é composta por agentes em busca de reconhecimento e notoriedade, principalmente por meio do capital científico objetivado, tendo como objetivo, inicialmente, a manutenção de sua posição na hierarquia científica. A visibilidade do agente inserido nesse espaço aumenta por meio de estratégias sociais e, também, por meio do acúmulo dos diferentes capitais, tais como: capital científico do tipo puro, capital científico do tipo institucional e também capital científico do tipo objetivado. Os diferentes pesquisadores presentes nesse espaço agem de acordo com as regras do espaço social impostas, na maioria das vezes, pelos dominantes que modificam "as leis de formação dos preços características do mercado universitário" e, assim, aumentam suas chances de lucro (BOURDIEU, 2013, p. 33). Com isso, os dominantes possuem algumas características:

[...] são os que conseguem impor a definição da ciência segundo a qual a melhor realização da ciência consiste em ter, ser e fazer aquilo que eles têm, são ou fazem. É por isso que deparamos sempre com a antinomia da legitimidade: no campo científico, tal como noutros, não há uma instância para legitimar as instâncias de legitimidade. (BOURDIEU, 2008, p. 91).

A construção do trabalho científico em determinado campo visa, portanto, estabelecer um conhecimento adequado para o funcionamento e dinamismo desse trabalho a partir das relações objetivas apresentadas pelos pesquisadores de produtividade em pesquisa em CI. Esse comportamento é influenciado também pelo capital incorporado; ou seja, a história de vida influencia toda a dinâmica de produção do conhecimento e, consequentemente, contribui para o fortalecimento da identidade do campo científico (BOURDIEU, 2013). 


\section{Conclusões}

Como conclusões, pode-se destacar que, em relação ao número de artigos em cada GT da ANCIB, associados às diferentes categorias/níveis dos pesquisadores de produtividade em pesquisa, no primeiro quinquênio 20052009, os artigos publicados por pesquisadores de produtividade em pesquisa de categorias/níveis 1A, 1C e 2 concentraram-se em valores relativos no GT-01, no GT-02 e no GT-02, respectivamente.

No segundo quinquênio, em relação ao número de artigos em cada GT da ANCIB associados às diferentes categorias/níveis dos pesquisadores de produtividade em pesquisa, identificou-se que os artigos publicados pelos pesquisadores de produtividade em pesquisa de categorias/níveis 1A, 1B e 1C se concentram em valores relativos no GT-07, no GT-02 e mais uma vez no GT02 , respectivamente. Isto sugere que alguns GTs passam a ocupar posição de maior visibilidade, especialmente nos últimos anos deste estudo.

Com os subsídios oferecidos anteriormente, responde-se à seguinte questão: como os artigos produzidos pelos pesquisadores de produtividade em pesquisa em CI contribuem para a construção do conhecimento científico no campo em estudo?

Nos Grafos que se referem às redes two-mode 2005 a 2009 e 2010 a 2014, respectivamente, estão apresentados os artigos agrupados segundo os temas dos GTs da ANCIB: no período de 2005 a 2009, o GT-02 é core do campo da CI, pois discute os principais aspectos que envolvem as teorias, metodologias e práticas científicas relacionadas à organização da informação e do conhecimento, e no período de 2010 a 2014, o GT-08 é o core do campo em questão, discutindo e sistematizando aspectos que envolvem a Informação e Tecnologia. Essa prevalência de artigos vinculados ao GT-08 pode estar relacionada com questões como o surgimento de novas bases de dados, softwares para tratamento e organização da informação, entre outras questões.

Em termos de relevância para o campo da CI, essa concentração ou não de artigos científicos em diferentes GTs da ANCIB significa a oportunidade de almejar ou não a manutenção e conservação de uma posição social significativa dentro do espaço social e relacional da CI, considerando os aspectos das 
relações objetivas inseridas no contexto de cada GT da ANCIB. Por isso, os pesquisadores de produtividade em pesquisa em CI, envolvidos nesse jogo de poder, têm interesses específicos definidos pela lógica do jogo; ou seja, sempre há conflitos, lutas, competição e disputas por troféus, por exemplo, e pelos melhores trabalhos, pela coordenação científica em cada GT da ANCIB e, consequentemente, pelo reconhecimento e notoriedade entre os pares. É fundamental que o pesquisador adote estratégias sociais e políticas de acordo com as dinâmicas do campo para acumular capital social, capital científico do tipo objetivado, puro e institucional, para almejar ou fazer a manutenção de sua posição social.

O capital (econômico, cultural, social, científico, entre outros) presente em determinado campo é uma espécie singular de capital simbólico, considerando seus efeitos em determinado contexto em que está inserido, tendo em vista o acúmulo do conhecimento. Essas especificidades estão ligadas à relação de poder, "[...] que funciona como forma de crédito, pressupõe a confiança ou a crença dos que o suportam porque estão dispostos (pela sua formação e pelo próprio facto de pertencer ao campo) a atribuir crédito" (BOURDIEU, 2008, p. 53).

A principal contribuição deste estudo é evidenciar os pesquisadores de produtividade em pesquisa em CI e agrupar os artigos produzidos por eles, segundo os temas dos GTs da ANCIB. Segundo o contexto da Sociologia da Ciência, "A situação de pesquisa é um mercado simbólico. Uma reunião [...], um Congresso, são mercados simbólicos sujeitos a mecanismos de manipulação de preços, conversões, negociações, etc.” (GIRARDI JUNIOR, 2007, p. 216).

Como recomendações, sugere-se compreender, na sua totalidade e complexidade, como a Sociologia de Pierre Bourdieu impregna as relações de construção da ciência como um todo, por meio de um processo de luta e de oposições, de competições e de contrapontos, abordado nesta pesquisa.

Às limitações da pesquisa pode ser incluída a dificuldade de apresentar todos os movimentos dos pesquisadores a partir mobilidade entre as diferentes categorias/níveis. 


\section{Agradecimentos} $(\mathrm{CNPq})$.

Ao Conselho Nacional de Desenvolvimento Científico e Tecnológico

\section{Referências}

ANCIB. Sobre. [S. l.], 2016. Disponível em: http://www.ancib.org.br/. Acesso em: 28 out. 2019.

BARATA, R. B.; GOLBAUM, M. Perfil dos pesquisadores com bolsa de produtividade em pesquisa do CNPq da área de saúde coletiva. Cadernos de Saúde Pública, Rio de Janeiro, v. 19, n. 6, p. 1863-1876, 2003.

BEN-DAVID, J. Introdução. In: BEN-DAVID, J. (org.). Sociologia da Ciência. Rio de Janeiro: FGV, 1975. p. 1-32.

BOURDIEU, P. Homo academicus. 2. ed. Florianópolis: Edufsc, 2013.

BOURDIEU, P. La spécificité du champ scientifique et les conditions sociales du progrès de la raison. Sociologie et Sociétés, Québec v. 7, n. 1, p. 91-118, 1975.

BOURDIEU, P. Les trois états du capital culturel. Actes de la recherche en sciences sociales, Paris, v. 30, n. 30, p. 3-6, 1979.

BOURDIEU, P. Os usos sociais da ciências: por uma sociologia clínica do campo científico. São Paulo: UNESP, 2004.

BOURDIEU, P. Para uma sociologia da ciência. Lisboa: Edições 70, 2008.

BOURDIEU, P. Questões de sociologia. Rio de Janeiro: Marco Zero, 1983.

BOURDIEU, P.; WACQUANT, L. J. D. Réponses: pour une anthropologie réflexive. Paris: Seuil, 1992.

BUFREM, L. S.; OLIVEIRA, E. F. T. de; SOBRAL, N. V. Produção científica sobre temas pertinentes ao GT 07 indexada na base de dados BRAPCI. In:

ENCONTRO NACIONAL DE PESQUISA EM CIÊNCIA DA

INFORMAÇÃO, 19., 2018, Londrina. Anais [...]. Londrina: ANCIB, 2018. p. 4592-4608.

CNPQ. Bolsas individuais no país. Brasília, 2017. Disponível:

http://cnpq.br/view/-

/journal_content/56_INSTANCE_0oED/10157/2958271?COMPANY_ID=1013

2. Acesso em: 28 out. 2019. 
CNPQ. Séries históricas até 2015. Brasília, 2015. Disponível em: http://cnpq.br/series-historicas/. Acesso em: 28 out. 2019.

CNPQ. [Site do CNPq]. Brasília: Ministério da Ciência, Tecnologia, Inovações e Comunicações, 2019a. Disponível em: http://www.cnpq.br/. Acesso em: 28 out. 2019.

CNPQ. Sobre a plataforma Lattes. Brasília, 2019b. Disponível em: http://lattes.cnpq.br/. Acesso em: 28 out. 2019.

FAPESP. Indicadores de Ciência, Tecnologia e Inovação em São Paulo. In: FAPESP. Análise da produção científica a partir de indicadores bibliométricos. São Paulo: FAPESP, 2005. p. 5-7.

GIRARDI JUNIOR, L. Pierre Bourdieu: questões de sociologia e comunicação. São Paulo: Annablume, 2007.

GUimarãeS, J. A. C.; GRACIO, M. C. C.; MATOS, D. F. O. Produção científica de bolsistas em pesquisa em Ciência da Informação do Conselho Nacional de Desenvolvimento Científico e Tecnológico (CNPq): um estudo com artigos de periódicos. DataGramaZero, Rio de Janeiro, v. 15, n. 2, p. 1-7, 2014.

LEITE, A. C. F.; ROCHA NETO, I. Perfil dos bolsistas de produtividade em pesquisa do CNPq em Educação. Revista Brasileira de Ensino Superior, Passo Fundo, v. 3, n. 4, p. 97-112, 2017.

LEITE, C. et al. Cenário e perspectiva da produção científica sobre competência em informação (CoInfo) no Brasil: estudo da produção no âmbito da ANCIB.

Informação \& Sociedade, João Pessoa, v. 26, n. 3, p. 151-168, 2016.

MELO, W. L.; BUFREM, L. S.; CORREIA, A. E. G. C. Bolsistas de produtividade em pesquisa 1 em Ciência da Informação: verificação da produtividade e percepções autorais em um ambiente de comunicação científica. In: ENCONTRO NACIONAL DE PESQUISA EM CIÊNCIA DA INFORMAÇÃO, 19., 2018, Londrina. Anais [...]. Londrina: ANCIB, 2018. p. 4360-4379.

MUELLER, S. P. M.; SANTANA, M. G. A ciência da Informação no CNPq: fomento à formação de recursos humanos e à pesquisa entre 1994-2002. DataGramaZero, Rio de Janeiro,, v. 4, n. 1, p. 1-19, 2003.

MUGNAINI, R.; CARVALHO, T. de; CAMPANATTI-OSTIZ, H. Indicadores de produção científica: uma discussão conceitual. In: POBLACION, D. A.; WITTER, G. P.; SILVA, J. F. M. da (org.). Comunicação \& produção científica: contexto, indicadores e avaliação. São Paulo: Angellara, 2006. p. 313-340. 
NORONHA, D. P. et al. Doutores egressos dos programas de pós-graduação em Ciência da Informação: temática das teses defendidas e atual vínculo docente. In: ENCONTRO NACIONAL DE PESQUISA EM CIÊNCIA DA INFORMAÇÃO, 8., 2007, Salvador. Anais [...]. Salvador: ANCIB, 2007. p. 112.

SACCO, A. M. et al. Perfil dos bolsistas de produtividade em pesquisa do CNPq atuantes em Psicologia no triênio 2012-2014. Psicologia, Brasília, v. 36, n. 2, p. 292-303, 2016.

WASSERMAN, S.; FAUST, K. Social network analysis: methods and applications. Cambridge: Cambridge University Press, 1994.

\title{
The Sociology of Bourdieu: evaluation of the scientific production of productivity researchers in Information Science
}

\begin{abstract}
The scientific field of Information Science is a hierarchical space of positions and is formed by objective relations. This study aims to understand the generation and construction of knowledge by researchers in Information Science with productivity grants in Brazil. Specifically, this study sets out to present Brazilian researchers in Information Science with research productivity grants and to group the different articles they have written, according to the themes of the Working Groups in the Brazilian National Association for Research and Post-Graduate Education in Information Science, known as ANCIB. The research sample consisted of a total of 47 researchers in Information Science with productivity grants, in the first five years from 2005 to 2009, and of 56 researchers in the second five-year period from 2010 to 2014. A total of 317 articles were published in journals in the first five years, and 597 in the second five-year period. These articles were used to make groupings based on the themes of the 11 Working Groups of the Brazilian National Association for Research and Post-Graduate Education in Information Science and to generate the two-mode networks by use of the Ucinet software. We have found that scientific work aims to establish adequate knowledge to operate its development from the objective relations presented by the researchers. We conclude that Brazilian researchers in Information Science with productivity grants involved in such a power game have specific interests defined by that game's mode of operation; that is, there is always competition for prizes, for the best papers, for scientific coordination, among other kinds of competition.
\end{abstract}

Keywords: Scientific field. Knowledge Construction. Working Groups of the ANCIB.

Recebido: 21/05/2019

Aceito: 22/11/2019 
${ }^{1}$ Fundamentado na tese de doutorado defendida em 6 de fevereiro de 2018 junto ao Programa de Pós-Graduação em Ciência da Informação (PPGCI) da Universidade Estadual Paulista Júlio de Mesquita Filho - Faculdade de Filosofia e Ciências - Câmpus de Marília, sob o título Sociologia de Pierre Bourdieu e os pesquisadores bolsistas de produtividade em pesquisa do CNPq em Ciência da Informação.

2 “O Currículo Lattes se tornou um padrão nacional no registro da vida pregressa e atual dos estudantes e pesquisadores do país, e é hoje adotado pela maioria das instituições de fomento, universidades e institutos de pesquisa do país." (CNPQ, 2019b, doc. não paginado).

${ }^{3}$ A rede two-mode envolve dois conjuntos de atores ou um conjunto de atores e um conjunto de eventos (WASSERMAN; FAUST, 1994). 